\title{
Staff Performance Appraisal using Fuzzy Evaluation
}

\author{
Nureize Arbaiy and Zurinah Suradi \\ Information System Department, Faculty of Information Technology \\ and Multimedia, University of Tun Hussein Onn Malaysia, Locked Bag \\ 101, Parit Raja, Batu Pahat, Johor, Malaysia \\ \{nureize, zurinah\}@uth.edu.my \\ WWW home page: http://fatekma.uthm.edu.my
}

\begin{abstract}
Most organizations use performance appraisal system to evaluate the effectiveness and efficiency of their employees. In evaluating staff performance, it usually involves awarding numerical values or linguistic labels to their performance. These values and labels are used to represent each staff's achievement by reasoning incorporated in the arithmetical or statistical methods. However, the staff performance appraisal may involve judgments which are based on imprecise data especially when human (the superior) tries to interpret another human (his/her subordinate) performance. Thus, the scores awarded by the appraiser are only approximations. From fuzzy logic perspective, the performance of the appraisee involves the measurement of his/her ability, competence and skills, which are actually fuzzy concepts that can be captured in fuzzy terms. Accordingly, fuzzy approach can be used to handle these imprecision and uncertainty information. Therefore, the performance appraisal system can be examined using Fuzzy Logic Approach and this was carried out in the study. The study utilized hierarchical fuzzy inference approach since performance evaluation comprises of four criteria; namely work achievement, skill knowledge, personal quality, and community services. The output of the study provides the ranking for staff performance. From this study, it is expected that reasoning based on fuzzy models will provide an alternative way in handling various kinds of imprecise data, which often reflected in the way people think and make judgments.
\end{abstract}

\section{Introduction}

Performance appraisal may be defined as a structured formal interaction between a subordinate and his/her superior. It usually embraces of a periodic interview, in which the work performance of the subordinate is examined and discussed. The appraisal also triggers to identify weaknesses and strengths as well as opportunities

Please use the following format when citing this chapter:

Arbaiy, N., Suradi, Z., 2007, in IFIP International Federation for Information Processing, Volume 247, Artificial Intelligence and Innovations 2007: From Theory to Applications, eds. Boukis, C., Pnevmatikakis, L., Polymenakos, L., (Boston: Springer), pp. 195-203. 
for improvement and skills development. In most cases, performance appraisal system is used by managers to evaluate the management of the effectiveness and efficiency of employees and/or other resources within the organization [1]. It is a tool that can create competitive advantages amongst employees. In the Malaysian Public Services, each staff is being evaluated through a performance appraisal system that was designed to be a systematic annual process which involved evaluating employee's set targets (SKT), perceived behavior evaluation and work achievement during the year of evaluation. Here, each departmental targeted activities, programs and projects were decided upon, in reference to predetermined overall organizational policy and strategies agreed upon at the beginning of the year of evaluation. And, the appraisal focuses upon the integration and achievement of individual targets, behavior and performance at work as compared to the goals of the organization.

Performance appraisals are mainly used for judgmental and developmental purposes [2] in order to make good administrative decisions. Performance measures are meant to provide more complete information about an entity's performance. Organizations rely on supervisors to sort out how well individuals under their supervisions perform. The hope is that supervisors can disentangle the effects of job changes, collective action, luck and their own likes and dislikes, to make an accurate judgment of how well individuals have performed over a period of time. However, this hope is rarely realized, the appraisers (or the supervisors) bring their own biases and information-processing problems to the task of performance appraisal, thus the appraisal are often flawed. Therefore, the evaluation given may involve information of uncertainty and subjectivity.

In most staff performance evaluation, the process usually involves awarding numerical values or linguistic labels to staff performance. These values and labels are used to represent the staff's achievement by reasoning using arithmetical or statistical methods. In general, those methods can be classified using nominal score and single numerical score. These numerical scores may refer to another numerical interval-value that refers to a certain category of achievement, which is equivalent to 100 percent value. However, in most cases, the evaluation of staff performance may be influenced by the appraiser's experience, sensitivity and standard(s). Thus, the scores awarded by the appraiser are only approximations and there is an inherent vagueness in the evaluation. However, if we looked into the evaluation using fuzzy logic approach, the performance of the appraisee involves the measurement of ability, competence and skills, which are fuzzy concepts that may be captured in fuzzy terms. Consequently, fuzzy logic approach can be implemented to manage the uncertainty information involved in performance evaluation.

This paper is divided into four sections. First, the introduction briefly describes the topic of this paper. Second, the approach and methods in this study were explained. This section includes some related work. The third section discusses the result of this study and the final section concludes the study. 


\section{Method}

Decision making is a process of problem solving that involves pursuing of goals under constraints. The outcome is a decision that should result in an action [3]. This is a difficult process due to the factors such as incomplete and imprecise information subjectivity, linguistics, which tend to be present to a lesser or greater degree. As knowledge involved in appraisal evaluation is an approximate and fuzzy logic has been successfully used for approximate reasoning in such cases, its application becomes significant to manage the uncertainty in the evaluation system.

The design of fuzzy system mainly involved two operations of knowledge base derivation and the selection of the fuzzy inference process to perform the fuzzy reasoning [4]. The successful development of a fuzzy model for a particular application domain is a complex multi-step process, in which the designer is faced with a large number of alternative implementation strategies [5]. Fuzzy logic addresses such applications perfectly as it resembles human decision making with an ability to generate precise solutions from certain or approximate information. The advantage of fuzziness dealing with imprecision fit ideally into decision systems; the vagueness and uncertainty of human expressions are well modeled in the fuzzy sets, and a pseudo-verbal representation, similar to an expert's formulation, can be achieved [6].

Fuzzy approach has been used to evaluate many type of performances such as product and marketing, finance, education and more. [7] suggest a new learning achievement evaluation strategy in student's learning procedure. That study aimed to overcome conventional approaches' shortcomings that can consider such vague factors as complexity, importance, and difficulty. [8] applied Fuzzy Multiple Criteria Decision Making to evaluate feasible developmental strategies in regard to the needs of manufacturers, government and consumers. In accordance, this study is also concerned with the Fuzzy Logic which mainly aims to manage the uncertainty information and human-like reasoning and approached the domain problem.

\subsection{Fuzzy System}

Fuzzy system generally involves three phases of fuzzification, fuzzy inference and defuzzification [9]. The SKT evaluation comprises of four criteria; namely work achievement, skill knowledge, personal quality, and community services. The fuzzy linguistic for these criteria chosen are identified as competent, need improvement, proficient or else. The input value is mapped into the membership function graph to obtain the confidence value of that particular input variable. The real value that is supplied into the system is converted to linguistic variables. Linguistic variables are assigned to several systems' input. Table 1 tabulates the linguistic variables for several system inputs. 
Table 1: Fuzzy Set Definition

\begin{tabular}{|l|l|l|}
\hline \multicolumn{2}{|c|}{ Fuzzy Variables } & \multicolumn{1}{c|}{ Fuzzy Set Linguistic Term } \\
\hline \multirow{4}{*}{ Input } & Work Achievement & $\begin{array}{l}\text { \{needs improvement, meet } \\
\text { expectation, , competent }\end{array}$ \\
\cline { 2 - 3 } & Skill Knowledge & $\begin{array}{l}\text { \{needs improvement, medium, } \\
\text { proficient }\end{array}$ \\
\cline { 2 - 3 } & Personal Quality & \{poor, fair, good\} \\
\cline { 2 - 3 } & Community Service & \{not active, average, fair\} \\
\hline \multirow{2}{*}{ Output } & Appraisal's Mark & $\begin{array}{l}\text { \{needs improvement, meet } \\
\text { expectation, exceed expectation }\end{array}$ \\
\hline
\end{tabular}

The elements of a fuzzy set are mapped to a universe of memberships values, $\mathrm{X}$ to the interval $[0,1][10]$ or $\mu_{A}(x): X \rightarrow[0,1]$. Membership function is denoted by $\left.\mu_{\mathrm{a}} \mathrm{X}\right)$. If $\mathrm{X}$ is a universe of membership values and $\mathrm{x}$ is an element of $\mathrm{X}$, then a fuzzy set $A$ is represented by $A=\left\{\left(x, \mu_{A}(x)\right)\right\}, x \in X$. For this system, several membership functions were drawn for corresponding variables. The $x$-axis represents the variable while the $y$-axis represents the confidence value ranges from 0 to 1.0 . The trapezoidal function was used to represent the linguistic variable.

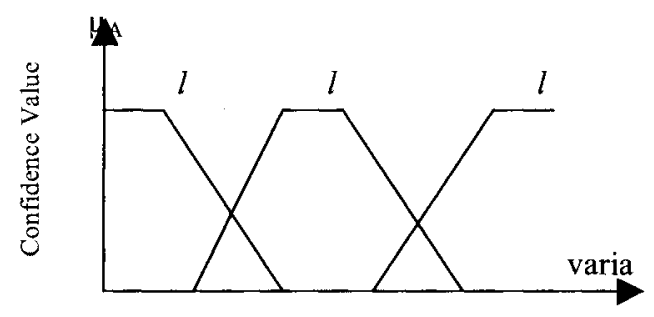

Figure 1: Trapezoidal Membership Function

From figure 1, $\Pi(\mathrm{x} 0, \mathrm{x} 1, \mathrm{x} 2, \mathrm{x} 3)=\left\{\begin{array}{cc}0 & x<x_{0} \\ \left(x-x_{0}\right) /\left(x_{1}-x_{0}\right) & x_{0} \leq x \leq x_{1} \\ 1 & x_{1} \leq x \leq x_{2} \\ \left(x-x_{2}\right) /\left(x_{3}-x_{2}\right) & x_{2} \leq x \leq x_{3} \\ 0 & x>x_{3}\end{array}\right.$

General trapezoid membership function graph drawn for this system variable is illustrated in Fig 1. From the membership function graph, a confidence value for each label will be retrieved from $\mathrm{x}$ - axis to $\mathrm{y}$-axis. 


\subsection{Fuzzy Inference}

Fuzzy inference engine resembles human reasoning in its use of approximate information and uncertainty to generate decisions. It consists of rules, facts and conclusions. The fuzzy production rules connect premises with conclusions, condition with action. In this inference, expert's knowledge and experience was acquired and formulate accordingly to develop the appropriate rule to perform the system. The fuzzy inference can be implemented using the if-then statements or Fuzzy Associative Memory (FAM). The if-then implementation is the same as that executed in expert system except that it involves the linguistic variables. Rules are simplified in fuzzy associative memory (FAM) tables to make the system easier to evaluate each set of rules. The single inference structure is shown as Fig 2-a. Therefore, as more than one attribute of inputs are involved in this system, then a hierarchical inference structure is needed (Fig.2-b).

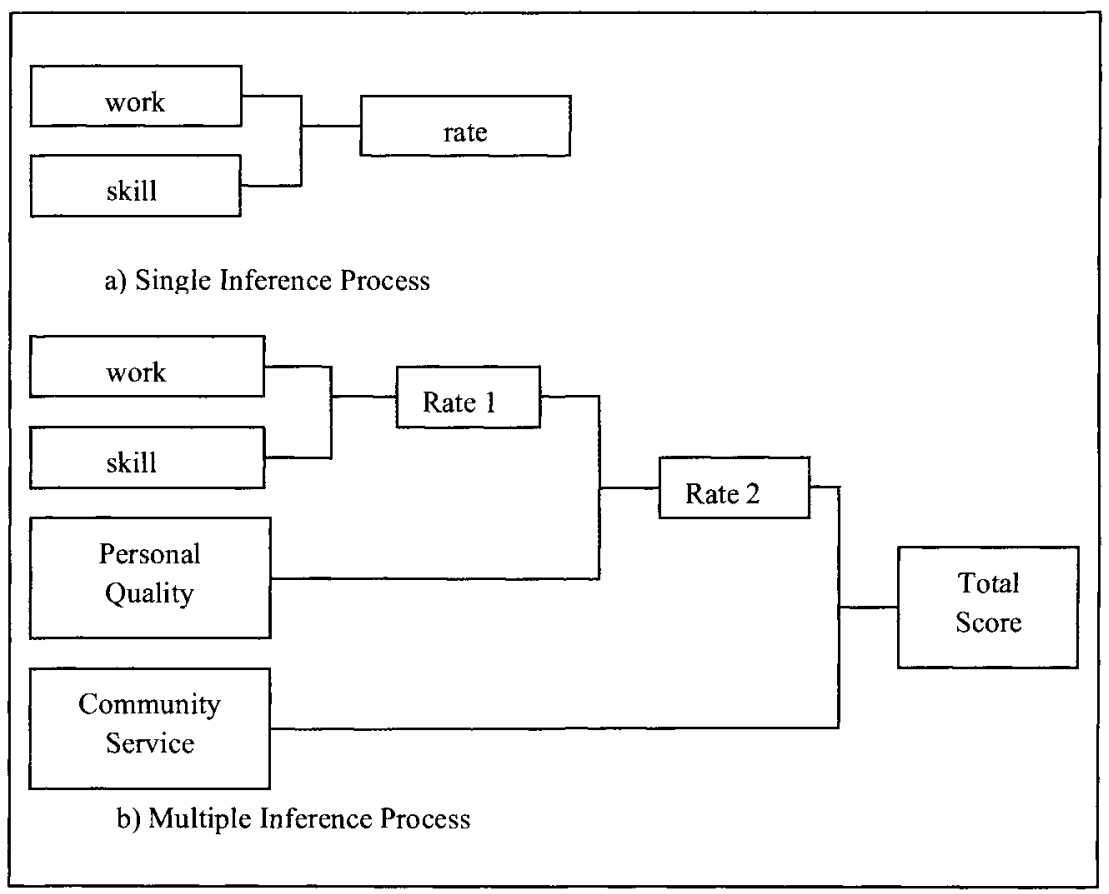

Figure 2: Fuzzy Inference Structure

Such fuzzy rules are represented as given below.

IF work_achievement is 'poor' AND skill is 'poor' THEN appraisal_rate is 'need improvement' 
IF work_achievement is 'poor' AND skill is 'satisfactory' THEN appraisal_rate is 'need improvement'

IF work_achievement is 'poor' AND skill is 'proficient' THEN appraisal_rate is 'meet expectation'

This fuzzy rule determines the decision embedded into the system engine, and should be validated from domain expert. Moreover, the human linguistic term involves in fuzzy rules enable the human-like fuzzy reasoning.

\subsection{Defuzzification}

Defuzzification or decomposition involves finding a value that best represents the information contained in the fuzzy set. The implementation of defuzzification is important in order to transform the linguistic terms back into crisp interpretation. The Defuzzification process yields the expected value of the variable for a particular execution of a fuzzy model. In fuzzy models, there are several methods of Defuzzification that describe an expected value for the final fuzzy state space could be derived [10].

There are a number of Defuzzification methods such as Centre of Gravity, Centre of Sums and Mean of Maxima. However, the system in this study only focuses on centre of gravity technique. The implementation of gravity technique is as modeled in eq 1 .

$$
\mu^{*}=\frac{\sum_{i=1}^{n} u_{i}^{*} \mu_{\text {out }}\left(u_{i}\right)}{\sum_{i=1}^{n} \mu_{\text {out }}\left(u_{i}\right)}
$$

The output is calculated as

$$
\text { output }=\frac{L w_{11}+L w_{12}+M w_{13}+L w_{21}+M w_{22}+M w_{23}+M w_{31}+M w_{32}+H w_{33}}{\sum_{w_{i}}^{9} i=1}
$$

Equation (2)

$\mathrm{H}$ represent as Excellent, $\mathrm{M}$ as meet_expectation and $\mathrm{L}$ as need_improvement respectively. In short, $\{\mathrm{H}, \mathrm{M}, \mathrm{L}\}$ are a fuzzy label (linguistic) used to represent the fuzzy variables. 


\section{Implementation and Results}

This section focuses on the implementation of Fuzzy Appraisal Evaluation System prototype and it is separated from the existing performance system. However it is assumed that the input parameter for this system is observed from the existing system. This application consists of fuzzy system components, which have been discussed above. The architecture of the system is illustrated as in Fig 3 .

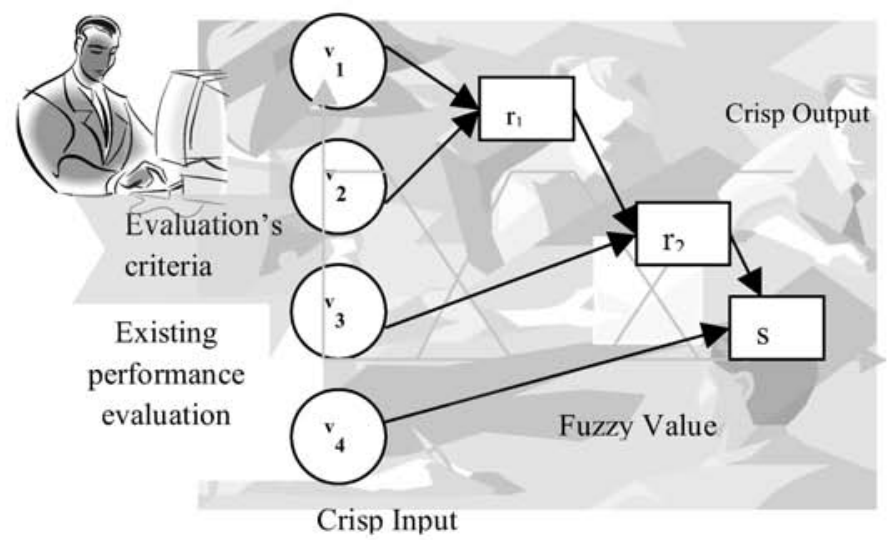

Figure 3: Fuzzy System Architecture for Appraisal Evaluation

This system uses four parameters as its system input. The input value actually should be observed from existing evaluation tool. However this study only focuses on the fuzzy system engine only, and assumed that the value is observed from the existing evaluation tool. So, it not does cover the details criteria for each group of input parameters. All inputs that are in the form of crisp value, will be processed throughout fuzzification phase, fuzzy inference and defuzzify the fuzzy value into crisp value for user presentation. As a prototype, this fuzzy system was developed using Microsoft Visual Basic (VB) 6.0 to test the fuzzy engine. Since VB provide easy programming facility, it was used as a platform to develop fuzzy engine as it consumed less time for coding task.

In practice, the 2-dimensional graph is used to represent a decision diagram using a total score accumulated from various criteria that be evaluated. The diagram can be illustrated in the Fig. 4 and is known as a singleton function. Consequently, the decision of forecasting staff's achievement will falls into specific conditions, which have a rigid boundary. Clearly the decision is based on crisp values which are rather rigid. 


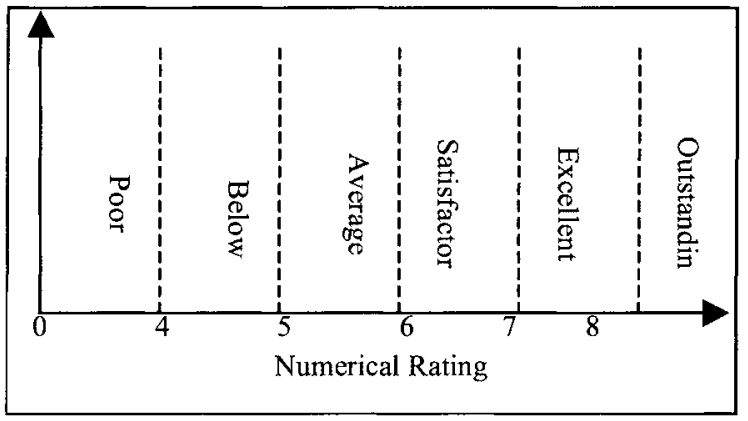

Figure 4: Example of Performance Decision

Based on the current practice, appraisal's evaluation for staff involves several criteria. Marks accumulated from the criteria then will be judge into single decision. For example, if the accumulated marks is above 85 than the staff will have an excellent result from the appraisal evaluation. In this study, several linguistic labels for each criterion are proposed. This study explores the evaluation process using more than one attributes. From the findings, if more than one attributes involved, the less rigid 3-dimensional decision graph was produced.

Although fuzzy logic eases the mapping problem, more programming effort was involved. To this end, fuzzy technique has definitely eases the mapping process. As a result, it produces more meaningful information to the user as well as improving the efficiency of handling uncertainty. Also, a fuzzy approach was used in order to accumulate the marks and assist the decision. Although fuzzy logic eases the mapping problem, more programming effort was involved. To this end, fuzzy technique has definitely eases the mapping process. As a result, it produces more meaningful information to the user as well as improving the efficiency of handling uncertainty.

However, the success of the system especially on the fuzzy engine relies much on the experience of selecting membership function and mainly the fuzzy knowledge base. The more expert knowledge involved and validated the rules and membership function, then the more reliable its end result. Thus, as expert systems rely on expert knowledge, fuzzy system also needs expert experience to strengthen the decision rules and capable to handle imprecise value in its reasoning.

\section{Discussion and Conclusion}

This study focuses on the implementation of fuzzy logic approach in the staff appraisal system. Therefore it emphasizes on the mapping of uncertainty data in performance measurement system into fuzzy values which consist of labels and 
confidence values. The mapping process is essential in this study since if erroneous membership function and rules were chosen, it may yield a flawed output. In future, it is urge that this system may be implemented in a web based version and incorporate with the existing evaluation system. Furthermore, others artificial intelligence techniques can be used in this study. For instance, expert system may be used to enhance the explanation facilities, or neural network technique to forecast the potential of staff performances. Finally, it is expected that reasoning based on fuzzy models will provide an alternative way in handling various kinds of imprecise data, which often reflected in the way people think and make judgments. It is important to point out that the aim of the proposed system is not to replace the current system of evaluating performance but it may be used to strengthen and improve the present system of evaluation by providing additional information for appraiser to make decision in performance evaluation and management of resources in an organization.

\section{References}

1. Armstrong, M. and Baron, A. (1998). Performance Management: The New Reality, London: Institute of Personnel and Development

2. Schweiger, I. and Sumners, G.E. (1994). Optimizing the Value of Performance Appraisals

3. Chan, D.C.K., Yung, K.L., Ip, A.W.H. (2002). An Application of fuzzy sets to process performance evaluation, Integrated Manufacturing Systems, 237-246.

4. Cordon, O., Herera, F., and Peregrin, A. (1999). Looking for the best Defuzzification method features for each implication operator to design accurate fuzzy model, Department of Computer Science and Artificial Intelligent, Spain.

5. Garibaldi, J.M. and Ifeachor, E.C. (1999). Application of simulated Annealing Fuzzy Model Tuning to Umbilical Cord Acid-base Interpretation, IEEE Transactions on Fuzzy Systems, Vol.7, No.1.

6. Hasiloglu, A.S., Yavuz, U., Rezos, S. and Kaya, M.D. (2003). A Fuzzy Expert System for Product Life Cycle Management, International XII, Turkish Symposium on Artificial Intelligence and Neural Networks.

7. Sunghyun, W and Jinil, $K$. (2001) Learning Achievement Evaluation Strategy Using Fuzzy Membership Function, 31 st ASEE/IEEE Frontiers in Education Conference

8. Tzeng, G.H., Teng, J.Y., Chang, S.L., and Lin, C.W. (2001) Fuzzy Multi-Criteria Evaluation Method for Developmental Strategies Of Hybrid Electric Vehicles, World Energy Council 18th Congress, Buenos Aires.

9. Zimmerman, H.J. (1996). Fuzzy Set Theory and Its Application, Third ed. Kluwer Academic Publishers, Boston, MA.

10. Nasution, H., (2002). Design Methodology of Fuzzy Logic Control, UTM 